\title{
Simulating deep convection with a shallow convection scheme
}

\author{
C. Hohenegger ${ }^{1, *}$ and C. S. Bretherton ${ }^{1}$ \\ ${ }^{1}$ Department of Atmospheric Sciences, University of Washington, Seattle, WA, USA \\ *now at: Max Planck Institute for Meteorology, Hamburg, Germany
}

Received: 10 December 2010 - Published in Atmos. Chem. Phys. Discuss.: 11 March 2011

Revised: 11 July 2011 - Accepted: 7 October 2011 - Published: 19 October 2011

\begin{abstract}
Convective processes profoundly affect the global water and energy balance of our planet but remain a challenge for global climate modeling. Here we develop and investigate the suitability of a unified convection scheme, capable of handling both shallow and deep convection, to simulate cases of tropical oceanic convection, mid-latitude continental convection, and maritime shallow convection. To that aim, we employ large-eddy simulations (LES) as a benchmark to test and refine a unified convection scheme implemented in the Single-column Community Atmosphere Model (SCAM). Our approach is motivated by previous cloud-resolving modeling studies, which have documented the gradual transition between shallow and deep convection and its possible importance for the simulated precipitation diurnal cycle.

Analysis of the LES reveals that differences between shallow and deep convection, regarding cloud-base properties as well as entrainment/detrainment rates, can be related to the evaporation of precipitation. Parameterizing such effects and accordingly modifying the University of Washington shallow convection scheme, it is found that the new unified scheme can represent both shallow and deep convection as well as tropical and mid-latitude continental convection. Compared to the default SCAM version, the new scheme especially improves relative humidity, cloud cover and mass flux profiles. The new unified scheme also removes the well-known too early onset and peak of convective precipitation over midlatitude continental areas.
\end{abstract}

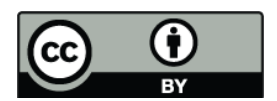

Correspondence to: C. Hohenegger (cathy.hohenegger@zmaw.de)

\section{Introduction}

Accurate representation of deep convection with global climate models of coarse resolution remains a nagging problem for the simulation of present-day and future climates. Typical biases include the simulation of a double Inter-Tropical Convergence Zone (ITCZ, see e.g., Bretherton, 2007; Lin, 2007), a too weak, too fast or spatially distorted Madden-Julian Oscillation (MJO, see e.g., Slingo et al., 1996; Bretherton, 2007) and poor timing of convection with a too early onset, peak and decay of precipitation. This last bias is apparent both over the Tropics (e.g., Yang and Slingo, 2001; Bechtold et al., 2004) and mid-latitude continental areas (e.g., Dai et al., 1999; Lee et al., 2007).

Many approaches have been proposed over the years to parameterize deep convection (see e.g., Arakawa, 2004; Randall et al., 2003, for a review). The most popular method remains the use of a mass flux scheme (see e.g., Plant, 2010; Arakawa and Schubert, 1974). The latter aims to predict the vertical structure and evolution of a one-dimensional entraining-detraining plume (bulk mass flux scheme) or spectrum thereof (spectral mass flux scheme). Irrespective of the specific design, convection schemes have to rely on some assumptions to relate the sub-scale cloud behavior to the large-scale resolved flow. Such relations are hard to get from observations and hard to formulate.

Recently, the use of large-eddy or cloud-resolving simulations to characterize the behavior of the cumulus ensemble has allowed the formulation of improved convective parameterizations. Rio et al. (2009) were able to simulate a realistic diurnal cycle of convection for an idealized case of mid-latitude continental convection by adding a density current parameterization to Emanuel (1991)'s convection scheme. Grandpeix et al. (2010) investigated this approach for the Hydrology-Atmosphere Pilot Experiment in the Sahel (HAPEX-Sahel) and the Tropical Ocean Global

Published by Copernicus Publications on behalf of the European Geosciences Union. 
Atmosphere Coupled Ocean Atmosphere Response Experiment (TOGA COARE) and found good agreement with cloud-resolving model simulations. Several studies also documented improvements in tropical convection, without nevertheless being able to fully remove the ITCZ or MJO biases, by employing more elaborate entrainment/detrainment formulations (e.g., Chikira and Sugiyama, 2010; Bechtold et al., 2008; Li et al., 2007; Wang et al., 2007), revised closures/triggering functions (e.g., Deng and Wu, 2010; Li et al., 2007; Zhang and Mu, 2005; Neale et al., 2008) or by introducing convective momentum transport (e.g., Deng and Wu, 2010; Richter and Rasch, 2008). The possible impacts of such modifications are in general strongly model dependent and confined to certain aspects of the simulated convection. In this respect it is still not clear whether a single convective parameterization can realistically handle both tropical oceanic and mid-latitude continental convection.

This study is geared towards improving the simulation of deep convection in coarse-resolution climate models. In contrast to the approach employed in most such models, we seek to develop a unified convection scheme starting from a parameterization designed for shallow cumulus convection. We regard shallow convection as mostly non-precipitating convection with no ice formation. Deep convection will refer to precipitating convection. Cloud-resolving modeling studies have documented the gradual transition occurring from shallow to deep convection and highlighted its importance for the simulated convective diurnal cycle (e.g., Guichard et al., 2004). This may be best achieved with a unified scheme. Our study is a step in this sense. We will explore how to unify shallow and deep convection and present singlecolumn model experiments to test our results.

The basic hypothesis behind our approach is that the main difference between shallow and deep convection is precipitation (both rain and snow) and its effects. Evaporation of precipitation (hereafter called rain evaporation) modifies the atmospheric environment and especially the structure of the planetary boundary layer (PBL), which feeds back on the convective development. Including such effects in a shallow convection scheme should thus allow the representation of deep convection within the same scheme. We thus see deep convection as highly interactive with the PBL state, like shallow convection. Our parameterization approach is further motivated by the results of recent large-eddy simulations (e.g., Khairoutdinov and Randall, 2006) which have highlighted the importance of rain evaporation for deep convection.

In order to fulfill our goals and test our hypothesis, we will employ large-eddy simulations of different convective events. We will investigate modifications in the PBL structure and in the atmospheric environment due to falling precipitation, and derive appropriate relations to describe them. These relations will then be implemented in the shallow convection scheme developed at the University of Washington (UW) by Bretherton et al. (2004) and Park and Bretherton
(2009). Using a single-column version of the National Center for Atmospheric Research (NCAR) Community Atmosphere Model (CAM), the performance of the new unified scheme will be assessed against large-eddy simulations, the default version of the CAM single-column model, and a version of the single-column model in which the UW shallow convection scheme is used without modification (but also without any separate deep convection scheme).

As this paper was being written, Mapes and Neale (2011) also presented results of CAM simulations with a unified convection scheme. They extended the UW shallow convection scheme to a two plume model and introduced a new prognostic variable called org to control the transition between shallow and deep convection. org is meant to represent convective organization and acts upon cloud-base properties and lateral mixing rates. The source of org is rain evaporation with an arbitrary set conversion rate. Our approach bears similarities with the one of Mapes and Neale (2011) as it also uses rain evaporation and its effects on cloud-base properties and mixing rates to control the transition from shallow to deep convection. However we stick to the one plume model, do not introduce new prognostic equations and employ largeeddy simulations to quantify the effect of rain evaporation on the subsequent cloud development.

The outline is as follows. Section 2 presents our method with a description of the different models, cases considered, and our experimental set-up. Section 3 focuses on the planetary boundary layer; changes in cloud-base mass flux and cloud-base thermodynamic properties between shallow and deep convection are investigated, parameterized and tested with single-column model experiments. Section 4 repeats the analysis for entrainment and detrainment rates. Conclusions are given in Sect. 5 .

\section{Method}

\subsection{Models}

The large-eddy simulations (LES) are performed with the System for Atmospheric Modeling (SAM, see Khairoutdinov and Randall, 2003). The model solves the 3D anelastic equations given prescribed large-scale tendencies and surface fluxes/sea surface temperature. As parameterization, the model includes a bulk microphysics scheme, a Smagorinskytype scheme to represent subgrid-scale turbulence, and the radiation package (Collins et al., 2006) taken from the NCAR CAM3 global climate model (GCM). A more detailed description of SAM can be found in Khairoutdinov and Randall (2003).

For the single-column model experiments we employ the Single-column (one-dimensional) version of the Community Atmosphere Model (SCAM, see Hack and Pedretti, 2000), version 3.5. SCAM comes with the full atmospheric parameterization package of the CAM3.5 GCM. This is a version 
of CAM3 (see Collins et al., 2006) with a modified treatment of deep convective momentum transport (Richter and Rasch, 2008) and a revised deep convective trigger (Neale et al., 2008). CAM3 includes a surface-driven boundary-layer turbulence scheme based on Holtslag and Boville (1993). Deep convection is parameterized after Zhang and McFarlane (1995) while shallow convection follows Hack (1994). As alternate parameterizations, the model can be run with new moist turbulence and shallow convection schemes developed at the UW (see Bretherton and Park, 2009; Bretherton et al., 2004; Park and Bretherton, 2009).

\subsection{The UW shallow convection scheme}

Since the UW shallow convection scheme serves as the starting point to develop a unified convection scheme, it is explained here in more detail. It is a mass flux scheme based on a buoyancy-sorting, entrainment-detrainment plume model. Updraft mass flux $M_{u}$ and updraft properties $\psi_{u}$ are computed according to:

$$
\begin{aligned}
\frac{\partial M_{u}}{\partial z} & =M_{u}(\epsilon-\delta) \\
\frac{\partial \psi_{u}}{\partial z} & =\epsilon\left(\bar{\psi}-\psi_{u}\right)+S_{\psi}
\end{aligned}
$$

with $\epsilon$ the fractional entrainment rate, $\delta$ the fractional detrainment rate, $\bar{\psi}$ the mean environmental property and $S_{\psi}$ source term. The mass flux at cloud base $M_{\mathrm{cb}}$ is determined by the ratio between convective inhibition (CIN) and mean planetary boundary layer turbulent kinetic energy (TKE):

$M_{\mathrm{cb}}=0.4 \rho \sqrt{\overline{\mathrm{TKE}}} \exp \left(-\frac{\mathrm{CIN}}{\overline{\mathrm{TKE}}}\right)$

with $\rho$ the air density. CIN is implicitly computed within the scheme (see Park and Bretherton, 2009), while TKE must be provided by the boundary layer scheme. This ensures tight interactions between the planetary boundary layer (PBL) and cumulus convection. If the lifting condensation level (LCL) is much higher than the top of the boundary layer, CIN is very large and air parcels don't have enough kinetic energy to overcome their CIN. The boundary layer height increases via entrainment until it reaches the LCL. As a result, CIN decreases, the mass flux increases and compensating subsidence increases preventing the PBL to rise further. The closure thus acts to keep the cumulus base near the top of the PBL and keeps CIN on the same order as TKE (Fletcher and Bretherton, 2010).

Cloud properties are expressed in terms of the total water mixing ratio $q_{\mathrm{t}}=q_{\mathrm{v}}+q_{1}+q_{\mathrm{i}}$ and the ice-liquid water potential temperature $\theta_{\mathrm{li}}=\theta-q_{1} L_{\mathrm{v}} / \Pi c_{p}-q_{\mathrm{i}} L_{\mathrm{f}} / \Pi c_{p}$ (Deardorff, 1976), with $\theta$ the potential temperature, $q_{\mathrm{i}}, q_{1}$ and $q_{\mathrm{v}}$ the ice, liquid water and water vapor mixing ratios, $L_{\mathrm{v}}$ and $L_{\mathrm{f}}$ the latent heat of vaporization and of sublimation, $c_{p}$ the specific heat of dry air at constant pressure, and $\Pi$ the Exner pressure function. Both $q_{\mathrm{t}}$ and $\theta_{\mathrm{li}}$ are assumed to be conserved for non-precipitating moist adiabatic processes. At cloud base, $q_{\mathrm{t}}$ is set to its surface value, while $\theta_{\mathrm{li}}$ is diagnosed from the lowest value of the virtual potential temperature over the PBL and the value of $q_{\mathrm{t}}$ at cloud base. Updraft vertical velocity $w_{u}$ is diagnosed solving

$\frac{1}{2} \frac{\partial}{\partial z} w_{u}^{2}=a B_{u}-b \epsilon w_{u}^{2}$

with $B_{u}$ updraft buoyancy, $a$ virtual mass coefficient and $b$ drag coefficient. $a$ and $b$ are set to 1 and 2, respectively (see Bretherton et al., 2004, for more detail). The updraft vertical velocity determines the maximum height reached by the plume.

Entrainment and detrainment processes are parameterized using buoyancy sorting principles. Mixing of cloudy air with environmental air generates a spectrum of mixtures with different buoyancies and vertical velocities. It is assumed that only mixtures that can travel a certain vertical distance $l_{\text {crit }}$ remain in the updraft. By assuming that the generated spectrum of mixtures is uniform, the fractional entrainment and detrainment rates per unit height are found to be

$$
\begin{aligned}
& \epsilon=\epsilon_{0} \chi_{\mathrm{c}}^{2} \\
& \delta=\epsilon_{0}\left(1-\chi_{\mathrm{c}}\right)^{2}
\end{aligned}
$$

The critical mixing fraction $\chi_{c}$ depends on height; at each level it is fully determined by the chosen $l_{\text {crit }}$ as well as by the updraft and environmental properties expressed by their buoyancy and humidity (see Eq. (B1) in Bretherton et al., 2004). The fractional mixing rate $\epsilon_{0}\left(\mathrm{~m}^{-1}\right)$ is set empirically to $8 / z$, with $z(\mathrm{~m})$ being the height above ground. The scheme also includes enhanced penetrative entrainment above the level of neutral buoyancy of the bulk updraft (see Eq. (D1) in Bretherton et al., 2004).

The UW shallow convection scheme employs extremely simple microphysics: condensate larger than $1 \mathrm{~g} \mathrm{~kg}^{-1}$ is removed from the updraft as precipitation, which is partitioned between a fixed fraction that can fall through the updraft (and which can only evaporate below the cumulus base) and a remainder that is detrained into the environment (and which can evaporate above cloud base). In either case, the evaporation rate depends upon the saturation deficit and the precipitation flux. Note that while rain evaporation drives organized downdrafts in reality, there is no explicit downdraft formulation in the scheme; evaporated precipitation homogeneously cools the entire grid cell.

In principle, the UW shallow convection scheme could be directly used to predict deep convection. It contains a representation of precipitation and ice formation processes as well as of evaporation. However, it does not include any feedback between falling precipitation and subsequent convective development, which, as stated in the introduction, might be important for deep convection. Within the framework of a bulk mass flux scheme, cloud-base mass flux, cloud-base properties and entrainment/detrainment rates are key quantities 
controlling the cloud development. Those are thus the three quantities that we will examine in more detail in Sects. 3 and 4 and modify with appropriate relationships to design a unified convection scheme.

\subsection{Cases}

In order to investigate issues related to the parameterization of moist convection, we consider three cases that have been well observed and extensively studied in the past. They have been chosen to span diverse atmospheric conditions and types of convection.

The first case is taken from measurements made at the Atmospheric Radiation Measurement (ARM) Southern Great Plains station between 19 June and 3 July 1997 (Julian days 170-186). This case typifies continental summertime midlatitude convection. The period encompasses a wide range of conditions, including clear days, shallow convection, diurnally forced convection, and precipitation associated with the passage of extratropical cyclones and fronts.

The second case represents tropical marine deep convection. The measurements are taken from the Kwajalein Experiment (KWAJEX) over the west Pacific warm pool. We restrict here our analysis to the period 24 July-10 September 1999 (Julian days 205-253).

Finally, we also consider the Barbados Oceanographic and Meteorological Experiment (BOMEX), a frequently simulated example of non-precipitating shallow trade-cumulus convection. The forcing data are derived from observations taken on 22-23 June 1969.

\subsection{Experimental set-up}

The three cases are simulated with SAM and with different versions of SCAM, using prescribed time-dependent profiles of large-scale vertical motion and horizontal advective heating and moistening as well as surface fluxes (for ARM) and sea surface temperature (for KWAJEX and BOMEX). Each SAM simulation is doubly periodic in the horizontal but employs a different grid. For the ARM case, SAM is run with a horizontal resolution of $500 \mathrm{~m}$ with $384 \times 384$ grid points and 96 vertical levels going up to $30 \mathrm{~km}$. The grid spacing varies between $50 \mathrm{~m}$ near the surface to $250 \mathrm{~m}$ in the midtroposphere. The KWAJEX simulation has a horizontal resolution of $1000 \mathrm{~m}$ and a vertical resolution of $100 \mathrm{~m}$ near the surface up to $400 \mathrm{~m}$ in the mid-troposphere. The domain contains $256 \times 256 \times 64$ grid points. For both ARM and KWAJEX, the domain-mean winds are nudged to the time-varying observational profiles with a one-hour relaxation time. Finally, the BOMEX simulation contains $256 \times 256 \times 96$ grid points with a resolution (both horizontally and vertically) of $40 \mathrm{~m}$. In the upper third of the domain, perturbations to the horizontal mean are linearly damped to help absorb convectively-forced gravity waves. For BOMEX, the winds are forced by a geostrophic wind profile rather than through nudging.

Similar simulations of SAM have been validated and investigated in detail by Khairoutdinov and Randall (2003) for ARM, Blossey et al. (2007) for KWAJEX, and Siebesma et al. (2003) for BOMEX. These studies show that the SAM model reproduces the overall convective development fairly accurately compared to observations in all three cases. Hence, we will use the SAM simulations as a benchmark both to characterize the behavior of the cumulus ensemble and to validate the SCAM single-column model experiments.

For all cases, SCAM is run with 30 vertical levels and a time step of $5 \mathrm{~min}$, driven by the same large-scale forcing and surface fluxes/sea surface temperature as SAM. For KWAJEX and BOMEX, the start and end times of the SCAM simulations coincide with the SAM integrations. For ARM, only specific rain events are simulated with SCAM instead of the full time period as a whole. This is to ensure that differences obtained between the integrations are due to the convective parameterization rather than to the simulation of different atmospheric conditions. Indeed, SCAM drifts away from SAM with time in ARM due mainly to different timings and amplitudes of individual rain events. For each rain event, we employ the SAM-simulated mean profiles as initial data for the SCAM simulations. The specific events that we simulate (see, e.g. Fig. 1) are days 174 (05:30 UTC Julian day (JD) 174 to 11:30 UTC JD 175), 176 (05:30 UTC JD 17611:30 UTC JD 177), 178 (05:30 UTC JD 178-05:30 UTC JD 179), 179 (05:30 UTC JD 179-05:30 UTC JD 180) and 180 (05:30 UTC JD 180-11:30 UTC JD 181). Days with strong large-scale forcing are omitted since SCAM will tend to perform well for those cases due to the use of prescribed largescale tendencies.

To investigate the performance of the new unified convection scheme, three main types of SCAM simulations are performed (Table 1). The first experiment employs the default version of the CAM3.5 model, in which PBL processes are parameterized after Holtslag and Boville (1993), shallow convection after Hack (1994) and deep convection after Zhang and McFarlane (1995). This simulation is called CAM and serves as our control experiment.

The second experiment employs the UW PBL scheme, the UW shallow convection scheme and no deep convective parameterization. In this case, precipitation associated with deep convection will only be produced if the full grid cell reaches saturation (through SCAM microphysical scheme) or if the shallow convection scheme by itself succeeds in producing deep plumes. It can thus be expected that this simulation will underestimate deep convection. The experiment is called UWS and is otherwise identical to the CAM experiment.

Finally, the last set of experiments uses the UW PBL scheme and a modified version of the default UW shallow convection scheme encompassing a unified treatment of shallow and deep convection. Otherwise the integrations 
Table 1. Overview of the different SCAM simulations. HB stands for Holtslag and Boville (1993), Hack for Hack (1994), ZM for Zhang and McFarlane (1995), UWPBL for the University of Washington PBL scheme (Bretherton and Park, 2009) and UW for the default University of Washington shallow convection scheme (Park and Bretherton, 2009). UWunif corresponds to the new unified convection scheme.

\begin{tabular}{lllllll}
\hline Name & PBL & Shallow Cu & Deep Cu & $\begin{array}{l}\text { Mass flux } \\
\text { Eq. (6) }\end{array}$ & $\begin{array}{l}\sigma_{q} \\
\text { Eqs. (7a, b) }\end{array}$ & $\begin{array}{l}\text { Entrainment } \\
\text { Eqs. (9-10) }\end{array}$ \\
\hline CAM & HB & Hack & ZM & & & \\
\hline UWS & UWPBL & UW & None & No & No & No \\
\hline UWSDall & UWPBL & UWunif & UWunif & Yes & Yes & Yes \\
UWSDpbl & UWPBL & UWunif & UWunif & Yes & Yes & No \\
UWSDe0 & UWPBL & UWunif & UWunif & No & No & Yes \\
UWSDe0mf & UWPBL & UWunif & UWunif & Yes & No & Yes \\
UWSDe0sq & UWPBL & UWunif & UWunif & No & Yes & Yes \\
\hline
\end{tabular}
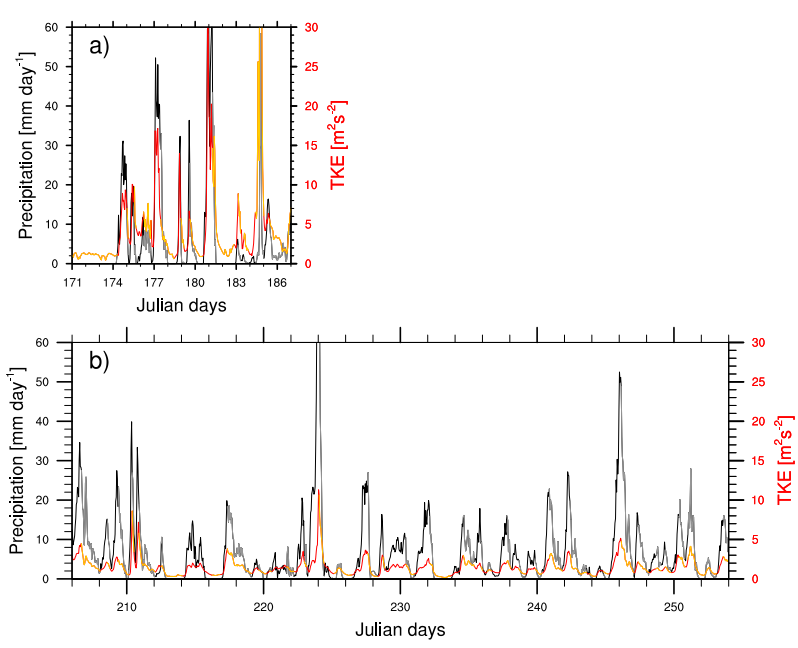

Fig. 1. Time series of precipitation at cloud base; black curve for onset and mature precipitation phase, grey for decay phase, and turbulent kinetic energy averaged over the planetary boundary layer; red curve for onset and mature phase; orange for decay phase, for (a) ARM and (b) KWAJEX.

are identical in their set-up to CAM and UWS. They are called UWSDpbl, UWSDall, UWSDe0, UWSDe0mf and UWSDe0sq, depending on the modifications made to the UW shallow convection scheme. The modifications are described along the text and in Table 1. Ideally, those simulations should stand in closer agreement to SAM than both the CAM and the UWS integrations.

\section{The planetary boundary layer under deep convection}

As stated in the introduction, we regard deep convection as shallow convection modified due to its production of heavy precipitation. In this view, the cloud-base mass flux in deep as well as shallow convection is regulated by the PBL and the subcloud mixed layer. Bulk instability measures like convective available potential energy (CAPE) are relevant to the vertical structure of cumulus convection, which in turn indirectly modifies the thermodynamic structure of the PBL and the overlying air. However, they are not viewed as direct controls on the cloud-base mass flux. This approach is supported by Kuang and Bretherton (2006), who showed that changes in CIN and TKE were closely correlated in largeeddy simulations of an idealized transition from shallow to deep convection and Fletcher and Bretherton (2010), who showed that a closure based on CIN and TKE could predict the cloud-base mass flux in LES simulations of ARM, KWAJEX and BOMEX. We especially refer to the study of Fletcher and Bretherton (2010) for more details on the advantages/disadvantages of employing a closure based on CIN and TKE. We nevertheless note that such a closure allows for a more straightforward implementation of precipitation effects on the cloud-base mass flux than a closure based on CAPE.

In this section, we thus investigate how changes in the PBL structure between shallow and deep convection, especially due to rain evaporation, affect cloud-base mass flux and cloud-base thermodynamic properties. Both are key parameters controlling the convective development. We use the SAM outputs to derive appropriate relations characterizing such effects. Except noted otherwise, all the quantities are computed from the SAM output statistics. The latter are computed at each time step and averaged both horizontally (if appropriate) and over one hour time interval. The derived relations are then implemented in the UW shallow convection scheme and tested in a single-column mode.

\subsection{SAM results}

\subsubsection{Cloud-base mass flux}

Figure 1 shows the time series of TKE and precipitation at cloud base $R R_{\mathrm{cb}}$ for ARM and KWAJEX obtained from the SAM output statistics (and thus based on hourly and 

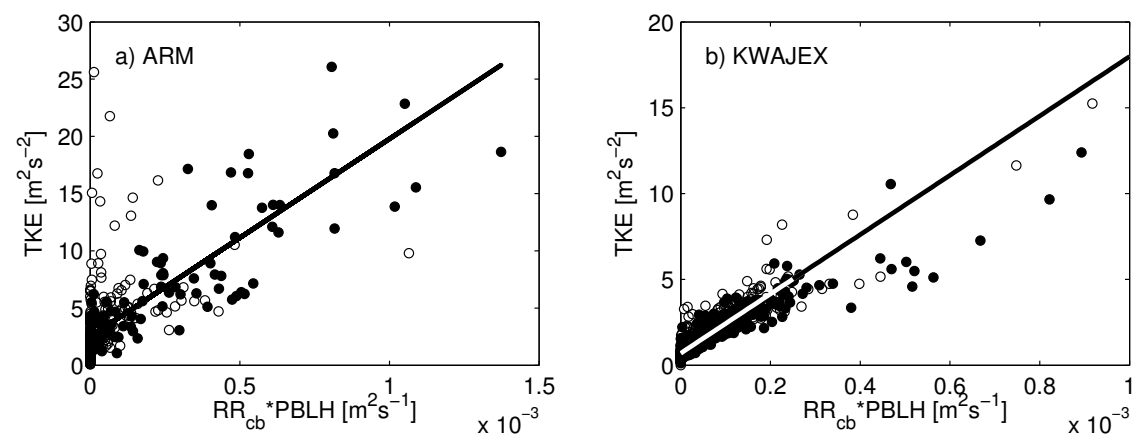

Fig. 2. Scatter plots of $\overline{T K E}$ versus $R R_{\mathrm{cb}}$. PBLH based on hourly statistics from (a) ARM and (b) KWAJEX. Full circles are for onset and mature precipitation phase, open circles for the decay phase. Onset, mature and decay phases are distinguished in Fig. 1. Slope of the solid regression line in (a) and (b) is $17280 \mathrm{~s}^{-1}$. There are 125 (530) and 285 (639) points in ARM (KWAJEX) for the onset/mature and decay phase, respectively.

horizontally averaged fields). TKE is averaged over the depth of the planetary boundary layer PBLH and is denoted hereafter TKE. PBLH is diagnosed as the height where the resolved-scale turbulent buoyancy flux reaches its minimum. The cloud base is defined following Fletcher and Bretherton (2010) as the lifting condensation level of an air parcel with a potential temperature equal to the potential temperature averaged over the layer $200-400-\mathrm{m}$ and a water vapor mixing ratio $q_{v}$ equal to the mean 200-400-m $q_{v}+\sigma_{q}$, where $\sigma_{q}$ is the horizontal standard deviation in $q_{v}$ averaged over the same height range. If the estimated cloud base is lower than the PBL height, we set its value to the height of the PBL, as done in the UW scheme.

It is evident in Fig. 1 that $\overline{\text { TKE }}$ increases from shallow to deep convection, i.e. with increasing precipitation. This increase is driven by rain evaporation, which generates cold pools that induce horizontal flows. Together with the associated organized surface convergence along cold pool boundaries, it represents a supplementary energy source for lifting an air parcel and thus favors the development of convection, as is apparent in our SAM simulations and many past studies of deep convection (see e.g., Rio et al., 2009; Khairoutdinov and Randall, 2006).

The increase in $\overline{\mathrm{TKE}}$ due to cold pool activity is not directly resolved by a coarse-resolution global model. Rio et al. (2009) represented this effect by implementing a density current parameterization and coupling it to Emanuel (1991)'s scheme. Here we follow a simpler, more empirical, approach to parameterize this effect.

Figure 2 shows a scatter plot of $\overline{\mathrm{TKE}}$ versus a measure of evaporative potential (and thus cold pool activity) formed as the product of $R R_{\mathrm{cb}}$ and PBLH, for our ARM and KWAJEX simulations. The full circles in Fig. 2 are for the onset/mature phase in which shallow convection is developing into deep precipitating convection, while open circles are for the decay phase. The times classified into the different phases, subjectively determined from the domain-mean precipitation time series, are indicated in Fig. 1 for reference.

Figure 2 indicates that TKE scales with $R R_{\mathrm{cb}} \cdot \mathrm{PBLH}$ with a similar slope both for KWAJEX and ARM. The value for zero precipitation should correspond to the TKE in a dry convective boundary layer $\overline{\mathrm{TKE}_{\mathrm{dry}}}$, which is predicted by the PBL scheme. We can thus write:

$\overline{\mathrm{TKE}}=\overline{\mathrm{TKE}_{\mathrm{dry}}}+C \cdot R R_{\mathrm{cb}} \cdot \mathrm{PBLH}$

with $C=17280 \mathrm{~s}^{-1}, R R_{\mathrm{cb}}$ in $\mathrm{m} \mathrm{s}^{-1}$, TKE in $\mathrm{m}^{2} \mathrm{~s}^{-2}$ and PBLH in $\mathrm{m}$. The correlation coefficient is 0.92 for KWAJEX and 0.83 for ARM during the onset/mature phase. The correlation is quite strong: adding further predictors does not provide any additional skill. The larger scatter in ARM results from the larger variability in the sampled synoptic conditions. The agreement worsens during the decay precipitation phase as cold pools need time to dissipate after rain evaporation is finished.

The evaporation of convective precipitation induces a positive feedback between convection and boundary-layer processes embodied in Eq. (6), because it generates TKE that yields more convection and more precipitation. However, rain evaporation also cools and stabilizes the PBL. At a certain point, the PBL collapses and shuts down convection. This effect is expressed by the use of PBLH in Eq. (6).

\subsubsection{Cloud-base thermodynamic properties}

Figure 3 shows example profiles of mass flux as a function of moist static energy (MSE) for ARM day 178 at 11:00 and 14:00 LT. In contrary to the other Figures, we employ the instantaneous 3D output from SAM to construct Fig. 3. 11:00 LT corresponds to the shallow convection phase, while 14:00 LT illustrates the situation under deep convection.

We use MSE as it is moist-adiabatically conserved and determines the temperature in saturated air. It is thus a useful and dynamically relevant characteristic of cumulus updrafts. This conservation is approximate in reality, but 

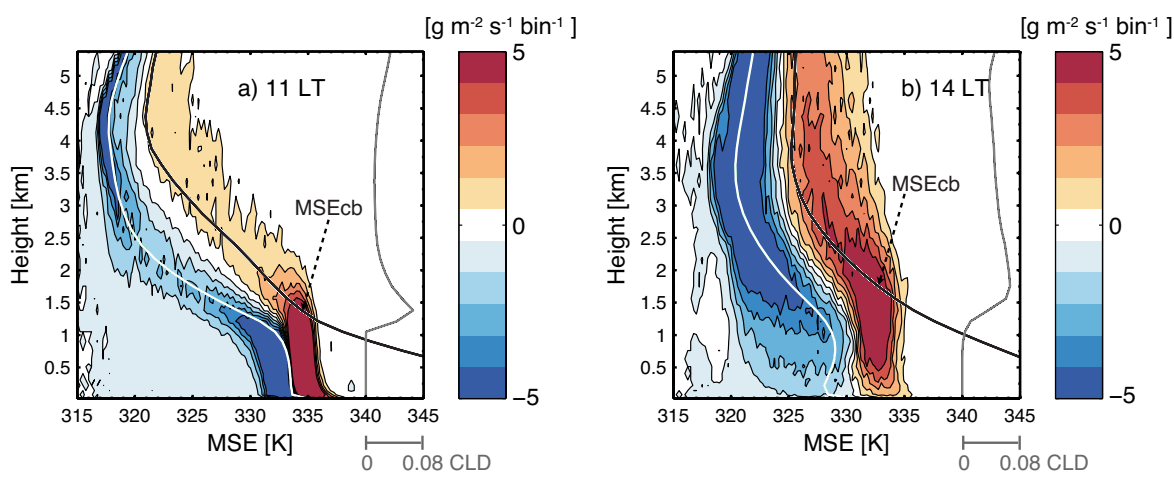

Fig. 3. Profiles of mass flux as a function of MSE for ARM day 178 at (a) 11:00 and (b) 14:00 LT (local time). White and black lines represent domain-averaged MSE (K) and saturation MSE (K). Grey line indicates the profile of cloud fraction (CLD), while the dashed arrow indicates $\mathrm{MSE}_{\mathrm{cb}}$.

is exact (except for ice processes) given the thermodynamic equations employed in SAM. Throughout this paper, MSE is rescaled into temperature units by dividing by $c_{p}=1006 \mathrm{~J} \mathrm{~kg}^{-1} \mathrm{~K}^{-1}$.

The profiles in Fig. 3 are obtained by binning at each height the grid points by their MSE and summing their mass flux per bin (see Kuang and Bretherton, 2006). The bin size is $0.25 \mathrm{~K}$. Light to dark red colors in Fig. $3 \mathrm{im}$ ply positive values of the vertical velocity and thus represent updrafts, while light to dark blue colors represent downdrafts/subsidence. Figure 3 also displays in white and black the domain-averaged MSE and the domain-averaged saturated MSE, as well as the domain-averaged cloud cover in grey. Equivalently, the shaded portion in Fig. 3 above the black line of the saturated MSE can be interpreted as representing the cloudy points.

Comparison of Fig. 3a and b reveals similarities and differences in the partitioning of cloud-base MSE between shallow and deep convective updrafts and downdrafts. The cumulus cloud base is visible in both plots as the altitude of maximum lower-tropospheric cloud fraction; at this level the mean updraft MSE, indicated by $\mathrm{MSE}_{\mathrm{cb}}$ in Fig. 3, is almost identical to the domain-mean saturation MSE at that height, suggesting the cumulus updrafts have nearly the same temperature (and hence buoyancy) as their environment at cloud base. Above cloud base, the net upward mass flux is carried almost exclusively within cumulus clouds. Since clouds are less numerous than cloud-free grid points the line of the domain-mean MSE does not pass in-between up- and downdrafts but is shifted towards the environment. The typical range of MSE carried by the upward mass flux is also vertically continuous across cloud base at both times.

Before strong precipitation (Fig. 3a), the PBL has a structure akin to the structure of a dry convective boundary layer. Half of the PBL experiences updrafts with slightly higher MSE, half downdrafts with slightly lower MSE and $\mathrm{MSE}_{\mathrm{cb}}$, as originating from the warmer part of the MSE spectrum, appears slightly warmer than the values of the domain-mean MSE in the PBL (the white line). Later on (Fig. 3b), precipitation-driven downdrafts bring a broad range of lower MSE into the PBL. Only the remaining high-MSE part of the PBL contributes to the convective cloud-base updrafts, and the difference between $\mathrm{MSE}_{\mathrm{cb}}$ and the values of the domainmean MSE in the PBL (the white line) increases.

We find that for both shallow and deep convection, the mean updraft MSE at cloud base $\mathrm{MSE}_{\mathrm{cb}}$ can be parameterized as follows (using SAM domain- and hourly-averaged statistics):

$$
\begin{aligned}
\operatorname{MSE}_{\mathrm{cb}} & =\overline{\operatorname{MSE}}+\left(L / c_{p}\right) \sigma_{q} \\
\sigma_{q} & =1 \times 10^{-3}\left(0.45+0.035 \min \left(R R_{\mathrm{cb}}, 20\right)\right)
\end{aligned}
$$

with $R R_{\mathrm{cb}}$ given in mm day ${ }^{-1}, \overline{\mathrm{MSE}}$ defined as the MSE averaged over the vertical layer $200-400 \mathrm{~m}, \sigma_{q}$ the horizontal standard deviation in specific humidity averaged over that same vertical layer and $L=2.5 \times 10^{6} \mathrm{~J} \mathrm{~kg}^{-1}$.

The expression in Eq. (7a) is inspired by Fletcher and Bretherton (2010), who, through trial and error, found it the most skillful at predicting cloud-base properties (see their Sect. 3a). Equation (7b) contains the approximation to compute $\sigma_{q}$. It is obtained by fitting a first-order polynomial in $R R_{\mathrm{cb}}$ to $\sigma_{q} . \quad R R_{\mathrm{cb}}$ is chosen as the predictor since the increased PBL variability is mainly due to cold pool formation. Note that, even without precipitation, Eq. (7a) will predict a small increase in $\mathrm{MSE}_{\mathrm{cb}}$. This is consistent with Fig. $3 \mathrm{a}$ and with the presence of turbulent eddies under shallow convection. Equation ( $7 \mathrm{~b}$ ) also sets an upper bound on $\sigma_{q}$ to express the fact that the pool of warm air available for updraft formation is limited, especially when cold pools begin to fill up the boundary layer.

The fit described by Eq. (7b) is illustrated in Fig. 4, using points from KWAJEX (full circles), ARM (open circles) and BOMEX (blue cross). Figure 4 indicates that Eq. (7b) is able 


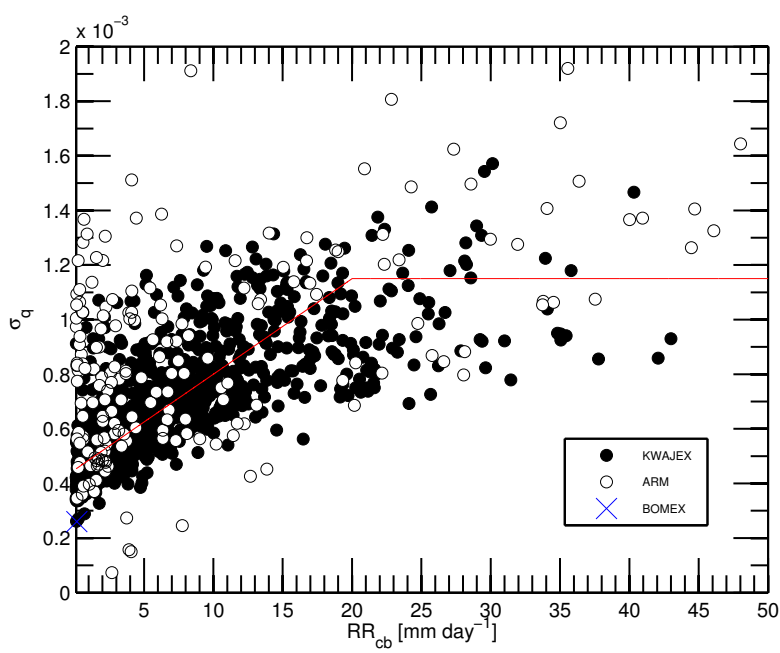

Fig. 4. Scatter plot of $\sigma_{q}$ versus precipitation at cloud base for KWAJEX (full circles, 1169 points), ARM (open circles, 410 points) and BOMEX (blue cross, 1 point) based on hourly statistics. The red line denotes the fit through the points (see Eq. 7b).

to capture the overall values of $\sigma_{q}$ for KWAJEX and ARM. As a numerical example, $\mathrm{MSE}_{\mathrm{cb}}$ is larger than $\overline{\mathrm{MSE}}$ by about $1 \mathrm{~K}$ on Fig. 3a, which matches the value of $\left(L / c_{p}\right) \sigma_{q}$ predicted by Eq. (7b) assuming no precipitation. The large spread by small precipitation amount, especially in ARM, is due to points from the decay phase where cold pools need time to dissipate. On the other hand, Eq. (7b) will overestimate $\sigma_{q}$ and $\mathrm{MSE}_{\mathrm{cb}}$ in BOMEX. Since this does not seem to negatively impact our results (see Sect. 4.2), use of a more complicated expression for $\sigma_{q}$ seems unwarranted.

\subsection{SCAM experiments}

We now use the results of Sect. 3.1 to modify cloud-base characteristics of the UW shallow convection scheme to help make it more suitable for deep convection. The new simulation is called UWSDpbl. In contrast to UWS, it employs the mass flux closure developed by Fletcher and Bretherton (2010) based on the same set of LES simulations as we use. This closure, like the default UW shallow cumulus mass flux closure, relates the mass flux to an exponential function of the ratio between CIN and TKE, but multiplies this function by a different prefactor. The closure reads:

$M_{\mathrm{cb}}=0.06 \rho w_{\mathrm{cb}} \exp (-\mathrm{CIN} / \overline{\mathrm{TKE}})$

$w_{\mathrm{cb}}=0.28 \sqrt{\overline{\mathrm{TKE}}}+0.64$

with $M_{\mathrm{cb}}$ mass flux at cloud base and $w_{\mathrm{cb}}$ velocity at cloud base. As an addition, UWSDpbl employs Eq. (6) to predict the cold pool contribution augmenting TKE in the mass flux closure Eqs. (8a)-(8b). The augmentation is done in the con- vection scheme, but similar results can be obtained by increasing TKE in the boundary layer scheme. This is because of the tight coupling existing between the two schemes when employing a CIN/TKE closure, as noted in Sect. 2.2. In UWS, TKE simply equals $\overline{\mathrm{TKE}_{\mathrm{dry}}}$, which is provided by the UW boundary layer scheme.

Cloud-base thermodynamic properties are expressed in UWSDpbl as the mean over the $200-400 \mathrm{~m}$ layer plus one standard deviation in humidity $\sigma_{q}$ (see Eq. 7a), instead of their surface or minimum values in UWS (see Sect. 2.2). $\sigma_{q}$ is predicted with Eq. (7b). Finally, the proportionality constant scaling the evaporation rate of falling precipitation is increased from $2 \times 10^{-6}$ to $1.5 \times 10^{-5}$ to be consistent with the values obtained from the SAM simulations (not shown).

It is important to note that the modifications in TKE and cloud-base thermodynamical properties introduced in UWSDpbl require PBLH and $R R_{\mathrm{cb}}$ as predictors. PBLH is passed over from the boundary layer scheme. For $R R_{\mathrm{cb}}$ we employ the precipitation averaged over the last hour to avoid undesirable effects associated with the on-off nature of convection schemes. The precipitation update also occurs at the end of the convection scheme and not in an iterative way. This prevents the scheme from adjusting within the loop rather than with time when transitioning from shallow to deep convection.

Figure 5 shows the diurnal cycle of precipitation for ARM days 176, 178, 179 and 180 for the simulations CAM, UWS, UWSDpbl and the SAM LES simulation. Day 174 exhibits similar features but is not included here for brevity. The default CAM configuration shows too weak a diurnal rainfall modulation that causes excessive morning precipitation. This problem is especially visible on day 178 , which constitutes the most archetypical example of surface forced convection during the period.

Both UWS and UWSDpbl better capture the timing of precipitation. The onset of precipitation coincides with SAM on days 176, 178 and 180 (Fig. 5a, b, d), while it is delayed on days 179 (Fig. 5c) and 174 (not shown). However, UWS and UWSDpbl also strongly underestimate the precipitation amounts. The cloud-base improvements in UWSDpbl increase the simulated amounts on day 178 but the impact remains generally small. This is understandable; the cloudbase improvements only affect the simulation of strongly precipitating convection; if the convection never produces significant rainfall, these improvements have no chance to modify the simulation.

Hence, the inclusion of precipitation-related modifications in cloud-base properties is insufficient to transform a shallow convection scheme into a realistic deep convection scheme. Analysis of the different days suggests that UWS and UWSDpbl have difficulties in transitioning to precipitating deep convection due to too large entrainment/detrainment rates. We address this problem in the next section. 

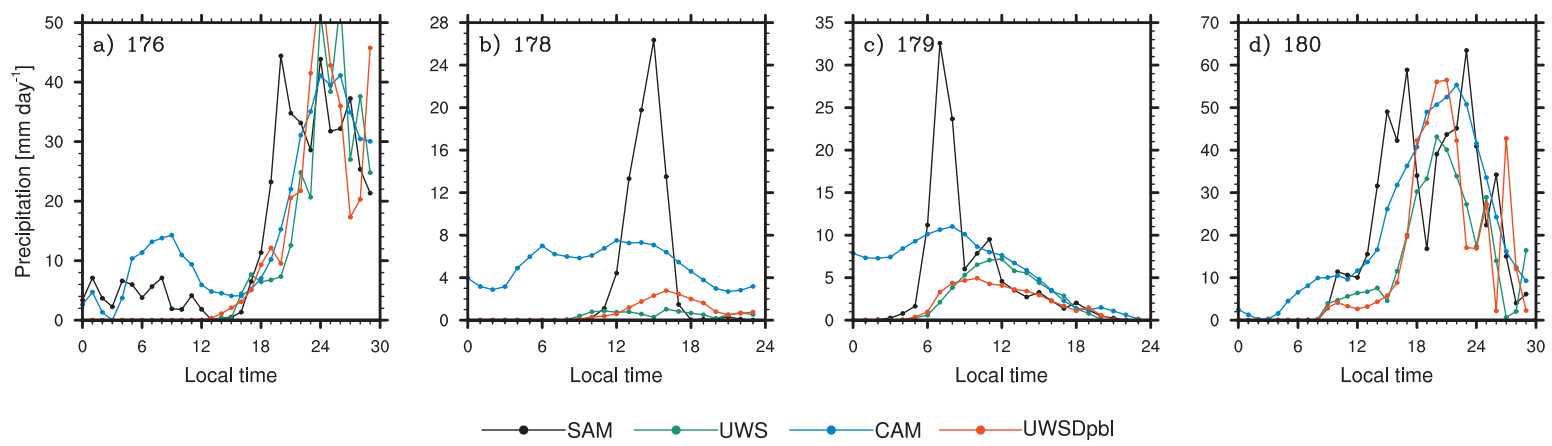

Fig. 5. Diurnal cycle of precipitation for ARM day (a) 176, (b) 178, (c) 179, and (d) 180. Black, green, blue and red lines are for SAM, UWS, CAM and UWSDpbl, respectively.

\section{Entrainment}

As in the previous section, we first employ the SAM simulations to derive formulations for entrainment and detrainment that work for both shallow and deep convection. We then implement and test them in combination with our cloudbase property modifications with single-column model experiments.

\subsection{SAM results}

Our approach retains the idea of buoyancy sorting described in Sect. 2.2, in which entrainment and detrainment rates are computed as $\epsilon=\epsilon_{0} \chi_{c}^{2}$ and $\delta=\epsilon_{0}\left(1-\chi_{c}\right)^{2}$ (i.e., Eqs. 5a and b), but SAM is used to revise the formulation of $\epsilon_{0}$.

In order to estimate $\epsilon_{0}$ from our SAM experiments, we first compute $\epsilon$ and $\delta$ using the equations for a simple plume model, as given in Eqs. (1) and (2) and done in previous LES studies. We sample all the cloudy points to compute the updraft mass flux and average it over one-hour time intervals. For the updraft property $\psi_{u}$, we choose the massflux weighted frozen moist static energy since it is approximately conserved ( $S_{\psi}=0$ ). The mass-flux weighted frozen moist static energy is again sampled over all cloudy points and hourly averaged, while $\psi$ corresponds to the domain and hourly averaged frozen moist static energy. Solving Eqs. (1) and (2) for $\epsilon$ and $\delta$, we can then compute $\epsilon_{o}$ from the buoyancy sorting relations $(5 a)-(5 b)$. This presupposes that entrainment and detrainment rates indeed follow buoyancy sorting principles.

The result of this procedure is shown in Fig. 6. Figure 6 shows as an illustration profiles of $\epsilon_{o}$ obtained for two different times in the KWAJEX simulation. The black solid line is associated with shallow cumuli with cloud tops reaching up to $2 \mathrm{~km}$. The red solid line is under deep convection. Figure 6 serves to illustrate that $\epsilon_{0}$ both varies with height and with the convective phase. At any given height, the values are larger during shallow cumulus convection. This is con-

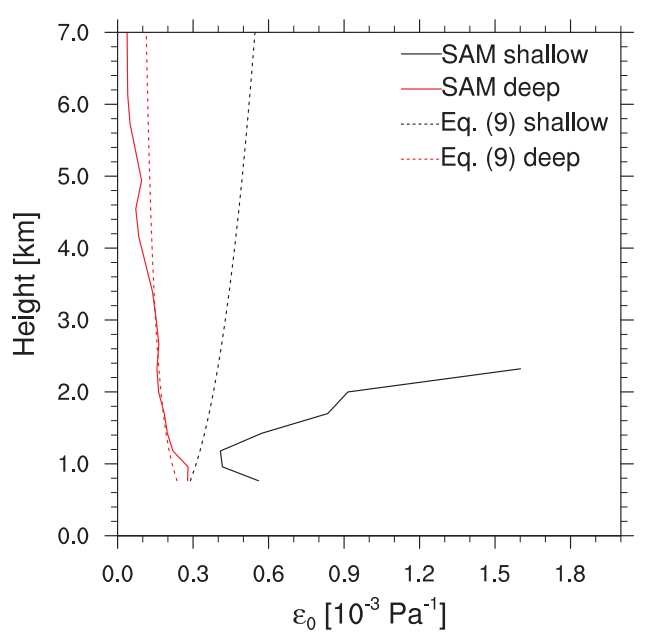

Fig. 6. Profiles of $\epsilon_{0}$ for two illustrative examples during KWAJEX: black line for the shallow phase, red line for the deep convection phase. The solid lines are from the SAM output, while the dashed lines are obtained using Eqs. (9) and (10).

sistent with previous LES studies (e.g Kuang and Bretherton, 2006; Khairoutdinov and Randall, 2006). Such studies have hypothesized that deep convective clouds, because of their larger size, entrain less than shallow cumuli.

Based on Fig. 6, the following generalized profile is used to diagnose $\epsilon_{0}$ :

$\epsilon_{0}(z)=\epsilon_{0}\left(z_{\mathrm{cb}}\right)\left(\frac{z}{z_{\mathrm{cb}}}\right)^{\alpha}$

with $z_{\mathrm{cb}}$ the height of the cloud base. $\alpha$ is implicitly computed by specifying $\epsilon_{0}$ at two "anchor" heights within the cumulus layer, namely the cloud base $z_{\mathrm{cb}}$ and a reference height $z_{1}$ that roughly corresponds to the minimum height of a cumulus updraft that will generate significant precipitation. 

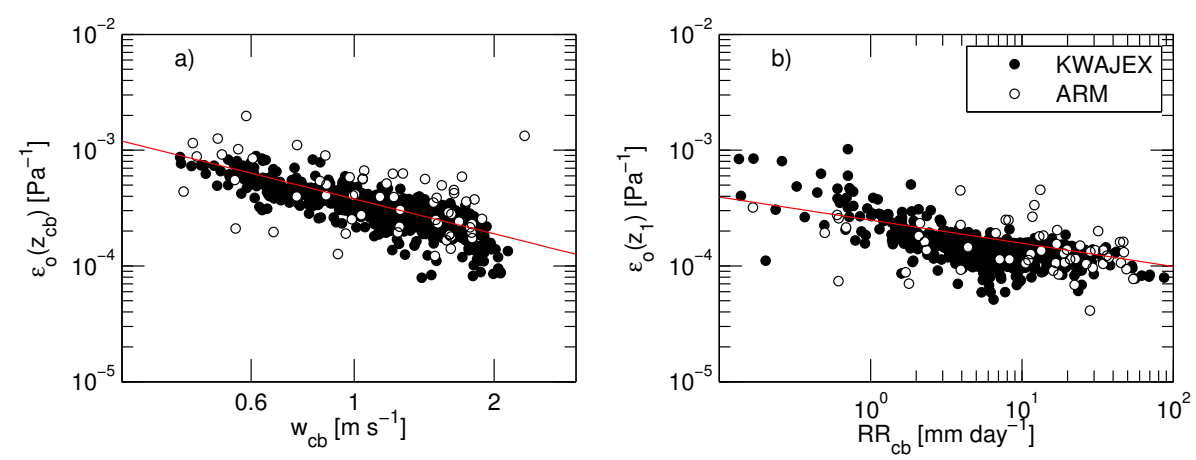

Fig. 7. Log-log scatter plots with regression line of (a) $\epsilon_{0}\left(z_{\mathrm{cb}}\right)$ versus $w_{\mathrm{cb}}$ and (b) $\epsilon_{0}\left(z_{1}\right)$ versus $R R_{\mathrm{cb}}$. Full circles for KWAJEX, open circles for ARM. Regression lines in (a) and (b) are given by Eqs. (10b) and (10c), respectively. Panel (a) uses the full hourly statistics output from KWAJEX and ARM where $R R_{\mathrm{cb}}>0.1 \mathrm{~mm} \mathrm{day}^{-1}$ and $0<\epsilon_{0}\left(z_{\mathrm{cb}}\right)<0.002 \mathrm{~Pa}^{-1}$. This corresponds to 1076 and 61 points in KWAJEX and ARM, respectively. Panel (b) only considers the onset and mature phase where $R R_{\mathrm{cb}}>0.1 \mathrm{~mm} \mathrm{day}^{-1}$ and $0<\epsilon_{0}\left(z_{1}\right)<0.002 \mathrm{~Pa}^{-1}$. This yields 482 and 59 points in KWAJEX and ARM, respectively.

The resulting relations read:

$$
\begin{aligned}
z_{1} & =z_{\mathrm{cb}}+2000 \mathrm{~m} \\
\epsilon_{0}\left(z_{\mathrm{cb}}\right) & =4.1 \times 10^{-3} /\left(\rho_{\mathrm{cb}} g w_{\mathrm{cb}}\right) \\
\epsilon_{0}\left(z_{1}\right) & =\exp (-8.3) \times\left(\max \left(R R_{\mathrm{cb}}, 0.1\right)\right)^{-0.2}
\end{aligned}
$$

In these formulae, $\epsilon_{0}$ is in $\mathrm{Pa}^{-1}, R R_{\mathrm{cb}}$ in $\mathrm{mm} \mathrm{day}^{-1}, w_{\mathrm{cb}}$ is the updraft velocity at cloud base $\left(\mathrm{m} \mathrm{s}^{-1}\right), \rho_{\mathrm{cb}}$ is air density at cloud base $\left(\mathrm{kg} \mathrm{m}^{-3}\right)$ and $g$ is gravity. The velocity at cloud base is computed from the SAM mass-flux weighted velocity, sampled at all cloudy points and hourly averaged. Our specific choice of $z_{1}$ is somewhat arbitrary; other choices can produce similar results as long as Eq. (10c) is appropriately adapted.

Figure 7 shows scatter plots supporting Eqs. (10b)-(10c). Beginning from Fig. $7 \mathrm{~b}$ and corresponding Eq. $(10 \mathrm{c}), \epsilon_{0}\left(z_{1}\right)$ is set proportional to the inverse of the precipitation at cloud base. The correlation coefficient amounts to 0.6. An upper bound, obtained in Eq. (10c) by setting $R R_{\mathrm{cb}}=$ $0.1 \mathrm{~mm} \mathrm{day}^{-1}$, is set on $\epsilon_{0}\left(z_{1}\right)$ to avoid large values for small precipitation amounts.

Covariability between $\epsilon_{0}$ and precipitation, as displayed by Fig. 7, is expected because higher precipitation amounts foster cold pool development which organizes the boundary layer. This produces larger and more coherent updrafts which have a lower bulk-mean entrainment rate, as noted above. Lower entrainment rates in turn favor the development of deeper clouds, hence sustaining a strong positive feedback between $\epsilon_{0}$ and $R R_{\mathrm{cb}}$.

Note that Fig. $7 \mathrm{~b}$ only includes the onset/mature precipitation phase, as marked in Fig. 1, to determine $\epsilon_{0}\left(z_{1}\right)$. During the decay phase, precipitation amounts are small, like in the onset phase, but mixing rates are small. Including those points in the regression reduces the slope of the regression line and results in too small mixing rates during the onset phase. This manifests itself by an overly rapid transition to deep convection in the single-column model experiments. The overestimation implied by Eq. (10c) for the decay phase does not seem to have any detrimental effect on the simulations.

At cloud base $\epsilon_{0}$ is chosen inversely proportional to the velocity at cloud base, as indicated in Fig. 7a and corresponding Eq. (10b). The correlation coefficient is 0.8 . We do not use $R R_{\mathrm{cb}}$ as a supplementary predictor since it does not add significant skill to this regression. Using $w_{\mathrm{cb}}$ is analogous to the approach of Neggers et al. (2002), who proposed $\epsilon=1 /\left(w_{u} \tau_{c}\right)$, where $w_{u}$ is the updraft velocity $\left(\mathrm{m} \mathrm{s}^{-1}\right)$ and $\tau_{c}=300 \mathrm{~s}$ is an empirical mixing timescale. In fact, our formulation would imply $\epsilon=4.1 \times 10^{-3} \chi_{c}^{2} / w_{\mathrm{cb}}$, which yields the same result for a typical cloud-base value $\chi_{c}=0.9$.

We also note that for values $w_{\mathrm{cb}}=0.5 \mathrm{~m} \mathrm{~s}^{-1}$ and $z_{\mathrm{cb}}=500 \mathrm{~m}$ typical of BOMEX, our formulation implies $\epsilon_{0}=$ $8 \times 10^{-3} \mathrm{~m}^{-1}=4 / z_{\mathrm{cb}}$, which is at the low end of the range of possible cloud-base values given in Table 1 of Park and Bretherton (2009) for the default UW scheme.

As a final illustration, the profiles of $\epsilon_{0}$ reconstructed by using Eqs. (9) and (10) and the SAM values for $\rho_{\mathrm{cb}}, w_{\mathrm{cb}}$ and $R R_{\mathrm{cb}}$, have been plotted as dotted lines in Fig. (6). Although not perfect, the fit captures the overall shape of the bulk entrainment rate profile and the corresponding difference between the shallow and the deep phase.

The formulation of $\epsilon_{0}$ in Eqs. (9)-(10) is admittedly empirical and tuned to our SAM simulations and to the way we computed it, which is a contentious issue by itself. It would be desirable in the future to use a more theoretically elegant approach tuned against a broader ensemble of simulations and observational constraints. However, our approach does try to build in some theoretically expected relationships between mixing rate and environmental variables and, as shown later, seems to produce plausible results. Equations (9)-(10) keep the essence of a bulk entrainment rate varying with height and implicitly with cloud size. The use 

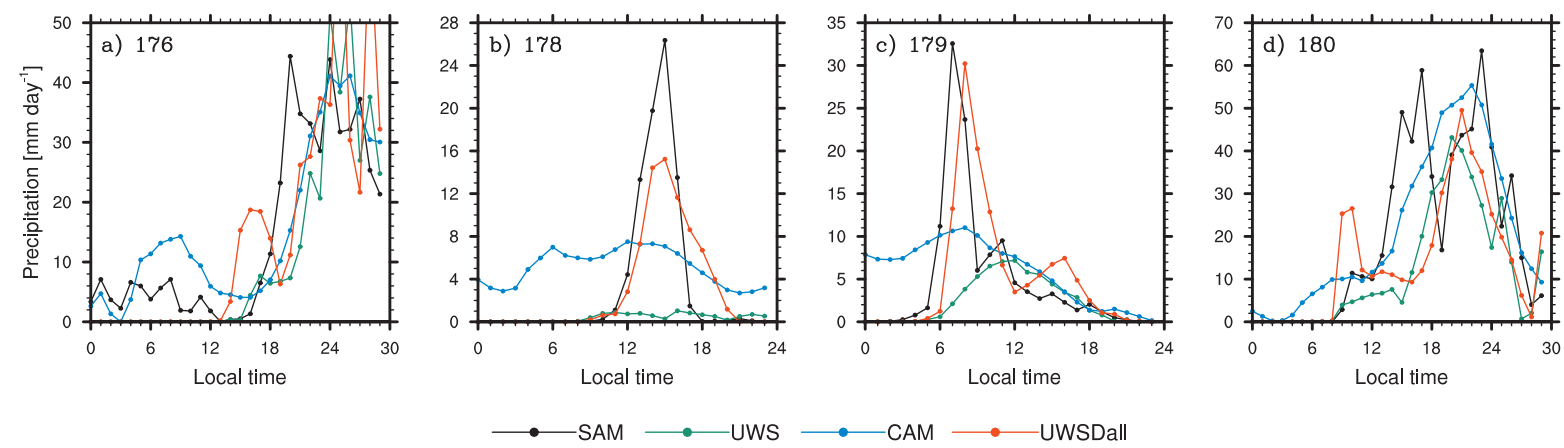

Fig. 8. Same as Fig. 5 but for SAM, UWS, CAM and UWSDall.

of precipitation at cloud base generalizes the specification of an inverse cloud radius as a predictor for entrainment rates (as in e.g. Kain, 2004) by allowing this radius to vary based on precipitation. Our approach can also produce similar results to decreasing entrainment rate at high ambient relative humidity, a method successfully applied by Bechtold et al. (2008), to the extent that higher environmental relative humidity will correlate with deeper clouds that yield more precipitation. Due to the strong feedback existing between entrainment and precipitation, there is obviously a causality issue. Given that removing rain evaporation has been shown to yield smaller clouds, larger entrainment rates and less precipitation (e.g. Khairoutdinov and Randall, 2006), there is some justification for using $R R_{\mathrm{cb}}$ as a predictor. This is also consistent with principles of organization (Mapes and Neale, 2011).

The main difference to entrainment/detrainment formulations currently applied in convective parameterizations is that Eqs. (9)-(10) do not require an explicit distinction between shallow and deep convection. Current formulations multiply their mixing rates by different prefactors. Here, through the production of precipitation and through changes in the environmental properties (as expressed by $\chi_{c}$ ), the mixing rates are allowed to vary with time and can support both shallow and deep convection. Equations (9)-(10) embody buoyancy sorting and organizational principles, which should apply to convection in general independently of the cloud depth. To which extent such a unified formulation can actually reproduce convection is investigated in the next section.

\subsection{SCAM experiments}

The revised entrainment-detrainment formulation is tested in SCAM by introducing it into UWSDpbl. As in UWSDpbl, we employ the precipitation averaged over the last hour as a predictor for $R R_{\mathrm{cb}} . w_{\mathrm{cb}}$ is diagnosed with Eq. (8b), while the other terms in Eqs. (9)-(10) are directly available. Two other changes are made to the default mixing scheme. First, no water is detrained before performing buoyancy sorting, as this tends to improve the results. Second, $\chi_{c}$ is limited to a maximum value of 0.5 above $6 \mathrm{~km}$ to avoid the development of instabilities due to compensating subsidence in cases of an increasing mass flux with height. The new simulation is called UWSDall (see Table 1).

Figure 8 shows the diurnal cycle of precipitation for ARM days 176, 178, 179 and 180 for UWSDall, CAM, UWS and SAM. Comparison to Fig. 5 reveals a strong impact of the new entrainment formulation. UWSDall produces stronger precipitation than UWSDpbl. The amounts are of comparable magnitude to the SAM simulation. Despite a tendency to produce too large precipitation amounts at the beginning of the onset phase, UWSDall clearly improves the simulated precipitation diurnal cycle as compared to CAM. This is especially true on day 178 (see Fig. 8b), where most convective parameterizations would fail (see Guichard et al., 2004).

UWSDall, in contrast to UWSDpbl, can realistically transition to deep convection. In principle, the moistening of the environment during the day through detrainment from previous shallow convection should increase $\chi_{c}$, so the mass flux decreases less rapidly with height and at some point significant mass flux reaches into the mid-troposphere. Nevertheless this effect did not appear sufficient in our singlecolumn model experiments, in contrast to results from cloudresolving studies (see especially Chaboureau et al., 2004). An additional and explicit sensitivity of fractional entrainment and detrainment rates to precipitation is required for the UW scheme to realistically transition from shallow to deep convection with the right diurnal timing.

Figure 9a-d shows cloud cover, mass flux (from the cloudy points), relative humidity and temperature profiles for UWSDall, CAM, UWS and SAM on day 178 averaged over the precipitation phase (10:00 to 18:00 LT). CAM simulates excessive cloud cover at all levels (see Fig. 9a) and an unrealistic mass flux profile (see Fig. 9b) compared to SAM. UWSDall underestimates the cloud cover above $2 \mathrm{~km}$. Since the computed cloud cover contains contributions from convective clouds, layered clouds and stratocumulus, where the cloud amount of the latter two categories is parameterized as 

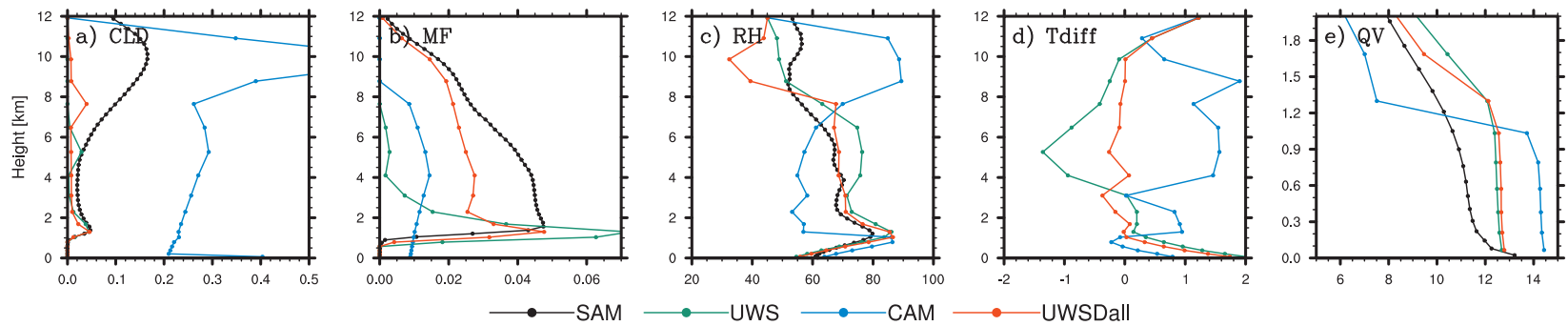

Fig. 9. Mean profiles of (a) cloud cover, (b) mass flux $\left(\mathrm{kg} \mathrm{m}^{-2} \mathrm{~s}^{-1}\right)$, (c) relative humidity (\%), and (d) temperature difference with respect to SAM (K) for ARM day 178. Lines as in Fig. 8. The profiles are averaged over the rain period, i.e., 10:00-18:00 LT. Panel (e) shows specific humidity $\left(\mathrm{g} \mathrm{kg}^{-1}\right)$ at 15:00 LT on ARM day 178 .
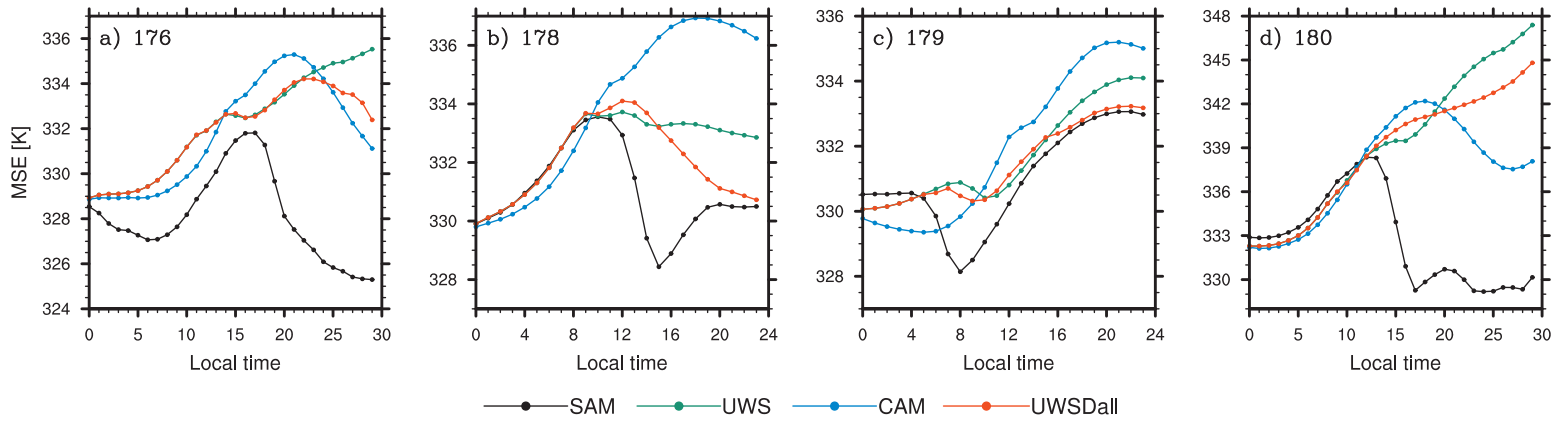

Fig. 10. Same as Fig. 8 but for MSE averaged over the lowest $1 \mathrm{~km}$.

a function of relative humidity, the observed underestimation is sensitive to the chosen relative humidity threshold for the onset of cloud formation. The mass flux profile in UWSDall is much more similar to SAM, with only a slight remaining underestimate of mass flux between 1.5 and $10 \mathrm{~km}$. This good agreement implies that the new entrainment formulation is able to capture typical entrainment and detrainment rate profiles in ARM. Similar conclusions hold for other times and ARM days.

In terms of relative humidity and temperature, Fig. 9c, $\mathrm{d}$ indicates that UWSDall outperforms CAM and UWS. The UWSDall curve tends to agree well with the SAM results. The relative performance of the simulations is casedependent. Significant improvements are obtained on days 178 and 179 (in which the diurnal cycle of surface fluxes is the main convective forcing) while all simulations perform similarly on the remaining days, on which large-scale advective forcing is more important (not shown).

One of the main biases of the simulations is visible in Fig. 9d and especially in Fig. 9e. Figure 9e shows specific humidity profiles at 15:00 LT, the time of maximum precipitation. CAM, UWSDall and UWS are all moister than SAM. They all exhibit a well-mixed boundary layer (see profile below about $1 \mathrm{~km}$ ), while SAM only remains well mixed in the upper part of the PBL (between about 300-900 m).
This bias is a fundamental consequence of the interaction of the boundary layer scheme with the deep convection. Both the UW PBL scheme and Holtslag and Boville (1993) do not consider horizontal heterogeneity within the boundary layer. To maintain convection, they must sustain a convective PBL that extends from the surface to the convective cloud base, or else the CIN will become too large to allow further cloudbase mass flux. The convective PBL must be nearly well mixed. On the other hand, the SAM humidity profile is due to cold pools in which moist, cool air spreads out along the surface in some parts of the domain, while updrafts are driven by surface fluxes and organized surface convergence in other parts of the domain. This does not mean that UWSDall does not feel the presence of cold pools. Cold pools only require spatially localized rain evaporation. Rain evaporation is present in the UW convection scheme and directly feeds back into the layer-mean temperature and moisture equations at each grid level, thereby affecting the PBL. Through the implemented relations, rain evaporation will also influence the development of moist convection. The resulting changes in convective activity will then feed back onto the PBL mainly through changes in PBL height (see Sect. 2.2). This again affects the mean PBL properties and the future development of convection. The feedback loop is thus consistent, but, due to the design of the PBL scheme, the full PBL has to uniformly 
respond to such changes.

Finally, Fig. 10 shows time series of MSE averaged over the lowest $1 \mathrm{~km}$ of the atmosphere, as a rough estimate for the PBL, for ARM. Figure 10 illustrates the other main deficiency of the single-column model experiments. All the SCAM simulations exhibit warmer MSE than SAM during the phase of heavy precipitation (compare to the precipitation time series in Fig. 8). The apparent missing stabilization of PBL MSE in SCAM is a direct consequence of not having explicit downdrafts in UWS and UWSDall. CAM does include downdrafts, but only saturated downdrafts. Yet most of the downdrafts appear to be unsaturated in SAM.

The absence of downdrafts in UWSDall does not preclude the use of Eqs. (6), (7), (9) and (10). Our approach recognizes that cold pools, whether created by subcloud evaporation as we can do in CAM, or created also by organized convective downdrafts as visible in SAM, affect the convective development. The fact that UWSDall can track precipitation and exhibits some reduction in MSE in Fig. 10 indicates that our modifications can indeed introduce a feedback between convective rainfall and changes in the boundary layer structure. The reported biases in MSE, especially towards the end of the different days, have no strong influence since we use prescribed large-scale forcing and simulate each day separately.

Figure 11 displays the results obtained for KWAJEX for the different simulations. We do not show precipitation since all the simulations perform well due to the use of a prescribed omega field. The different profiles in Fig. 11a-d have been averaged over the full time period. As in ARM we can recognize the improvements in the simulated cloud cover and mass flux profiles in UWSDall as compared to CAM and UWS. UWSDall also captures the relative humidity profile very well, while both CAM and UWS tend to overmoisten the troposphere, especially above 3 and $1 \mathrm{~km}$, respectively. Finally, no strong biases can be detected in the simulated temperature profile in UWSDall.

As in ARM, Fig. 11e reveals the bias toward a well-mixed PBL in the SCAM simulations. CAM and UWSDall appear too cold and too dry, while they were too warm and too moist in ARM (Fig. 9d, e). Time series of mean PBL MSE (not shown) reveals that the depletion of MSE in CAM and UWSDall during the precipitating phase is similar both in ARM and KWAJEX. Since the depletion is much stronger in SAM in ARM than in KWAJEX due to stronger downdrafts, this results in a warm and moist (cold and dry) bias in ARM (KWAJEX). We thus conclude that the ventilation of the PBL is too strong in UWSDall, which partly compensates for the missing downdrafts. In opposition, UWS never exhibits a strong depletion in MSE and thus is characterized by a warm and moist bias in all the cases.

Finally, the results for BOMEX are displayed in Fig. 12 with profiles of liquid water potential temperature, total specific humidity, cloud cover and mass flux for UWS, CAM, UWSDall and SAM. The profiles have been averaged over hours 3 to 6 of the BOMEX integrations, as in Park and Bretherton (2009). CAM exhibits similar biases to those noted in Park and Bretherton (2009) with excessive cloud cover throughout the cumulus layer. This bias is mainly removed in UWS and UWSDall. Although differences exist in the simulated profiles between UWS and UWSDall in Fig. 12, UWSDall is still able to simulate a typical case of shallow convection as well as UWS. In particular, with UWSDall, as with UWS, the simulated clouds remain shallow. Employing the Zhang and McFarlane (1995) scheme as the sole convective parameterization in CAM would erroneously simulate some deep convection for BOMEX.

Hence in terms of large-scale variables UWSDall agrees well with SAM in many respects. It provides improved single-column simulations of tropical oceanic, mid-latitude continental and shallow convection than the default version of the CAM model. It also gives more realistic simulations than UWS of both deep convection cases.

\subsection{Sensitivity}

In the previous section, we demonstrated that UWSDall compares better to SAM than either CAM or UWS. However, it remains to be shown whether all the included modifications are important for these improvements. From the results in Sect. 3 it is clear that the mixing rates need to be reformulated. The necessity of the changes in cloud-base mass flux and cloud-base thermodynamic properties are investigated in this section.

To that aim we perform three sensitivity experiments called UWSDe0, UWSDe0mf, and UWSDe0sq (see Table 1). UWSDe0 is identical to UWSDall except that it only includes entrainment/detrainment effects, not the modifications to $\overline{\mathrm{TKE}}$ (Eq. 6) and thermodynamic properties (Eqs. 7a, b). UWSDe0mf and UWSDe0sq build on UWSDe0: UWSDe0mf adds only the changes in cloud-base mass flux via changes in TKE (Eq. 6), while UWSDe0sq adds only the changes in cloud-base thermodynamic properties (Eqs. 7a, b) via changes in $\sigma_{q}$.

Figure 13 shows the corresponding time series of precipitation for the ARM days 176, 178, 179 and 180. The differences between UWSDe0, UWSDe0mf and UWSDe0sq are larger on days 178-179, which are dominated by surface flux forcing, than on days 176 and 180 (and in the KWAJEX simulation), which have stronger advective forcing. All simulations initiate convection at the same time, which is expected since both cloud-base changes only affect the parameterization when there is already convective rainfall. However for days $178-179$, all three new cases produce a period of rainfall with too weak a maximum and lasting too long compared to both SAM and UWSDall. We conclude that both cloudbase changes are required to make a sufficiently strong feedback between convective rainfall and changes in the boundary layer structure. 

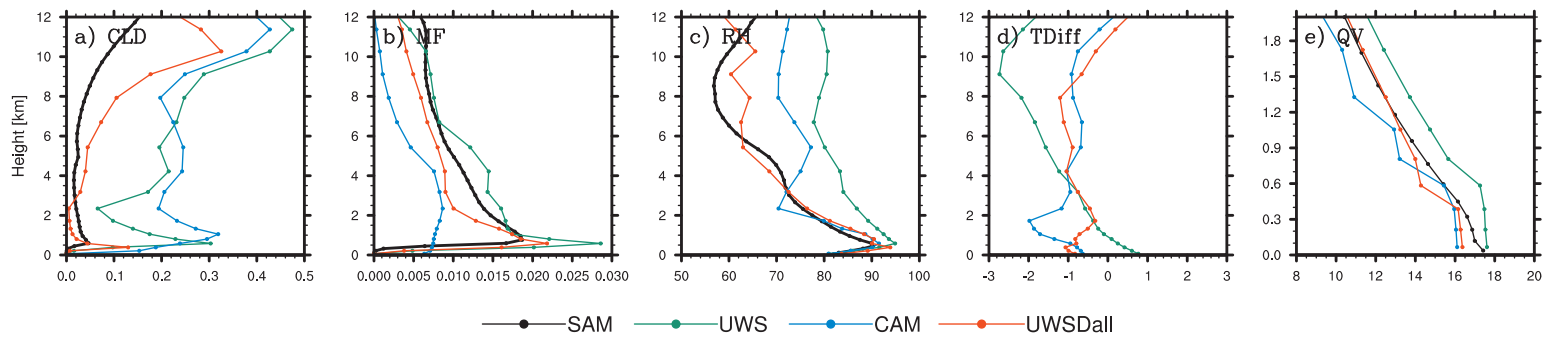

Fig. 11. Same as Fig. 9 but for KWAJEX. The profiles in (a)-(d) have been averaged over the full time period, while panel (e) displays a specific time under strong precipitation (hour 230 in the simulation).
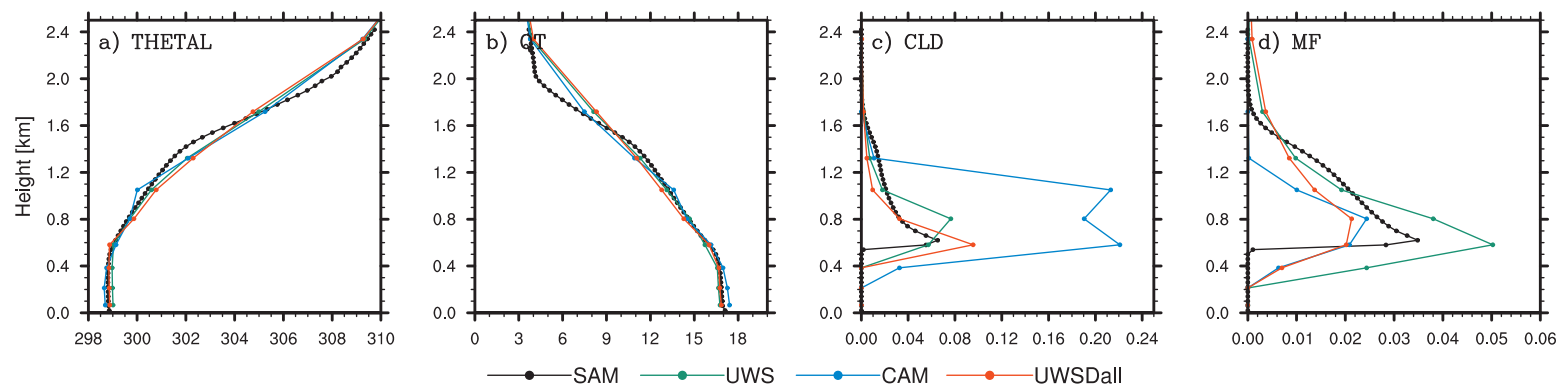

Fig. 12. Profiles of (a) liquid water potential temperature $(\mathrm{K})$, (b) total specific humidity $\left(\mathrm{g} \mathrm{kg}^{-1}\right)$, (c) cloud cover and (d) mass flux $\left(\mathrm{kg} \mathrm{m}^{-2} \mathrm{~s}^{-1}\right)$ averaged over hours 3 to 6 of BOMEX, for the same simulations as in the previous figures.

The increase in precipitation in UWSDe0mf and UWSDe0sq versus UWSDe0 follows from an increased mass flux at all heights. This stands in better agreement to the SAM values (not shown). The enhanced mass flux in UWSDe0 $\mathrm{mf}$ is a direct consequence of both enhanced cloud-base mass flux and more frequent triggering of convection, as expected from Eq. (6). The enhanced mass flux in UWSDe0sq follows from an enhanced entrainment rate and decreased detrainment rate at cloud base, which thus allow more plumes to be retained in the updraft. The latter changes in $\epsilon$ and $\delta$ relate to a value of $\chi_{c}$ larger in UWSDe0sq than in UWSDe0, as expected from the use of moister updraft parcels.

For most other variables, the differences between UWSDe0mf, UWSDe0sq and UWSDe0 are small, both in ARM and KWAJEX. The exceptions are of course the TKE values and the cloud-base thermodynamic properties.

Figure 14 displays scatter plots of TKE in SCAM versus SAM for the ARM, KWAJEX and BOMEX cases. On the left, we show UWS as an example for the simulations which do not include the TKE increase due to cold pool activity (i.e., UWS, UWSDe0, UWSDe0sq). On the right, UWSDall is chosen as an example for the two remaining simulations, where Eq. (6) is used.

As indicated by Fig. 14 and as expected, $\overline{\text { TKE }}$ is strongly underestimated in UWS (or equivalently UWSDe0 and
UWSDe0sq), while UWSDall (and UWSDe0mf) are in better agreement with SAM. The latter two simulations are able to capture the increase in TKE during precipitation events and thus confirm the appropriateness of Eq. (6). The overall underestimation in Fig. 14b is due to a slight underestimation of the boundary layer height in UWSDall. The points where a strong discrepancy between SCAM and SAM values remains visible in Fig. 14b correspond to those times where UWSDall produces no or only weak precipitation, while SAM records strong precipitation.

In terms of cloud-base thermodynamic properties, the use of Eq. (7b) yields an increase in cloud-base MSE. This increase amounts to up to $2 \mathrm{~K}$ in UWSDe0sq (and UWSDall) with respect to UWSDe0 (or UWS, UWSDe0mf). Given the existing biases in the PBL (see Sect. 4.2) this agrees better with SAM for KWAJEX, but less well for ARM.

\section{Conclusions}

This study has aimed to improve the simulation of deep convection with coarse-resolution climate models. Our specific goal has been to develop and assess the suitability of a unified convection scheme, capable of handling both shallow and deep convection. Our approach is based on the hypothesis that the main difference between shallow and deep 

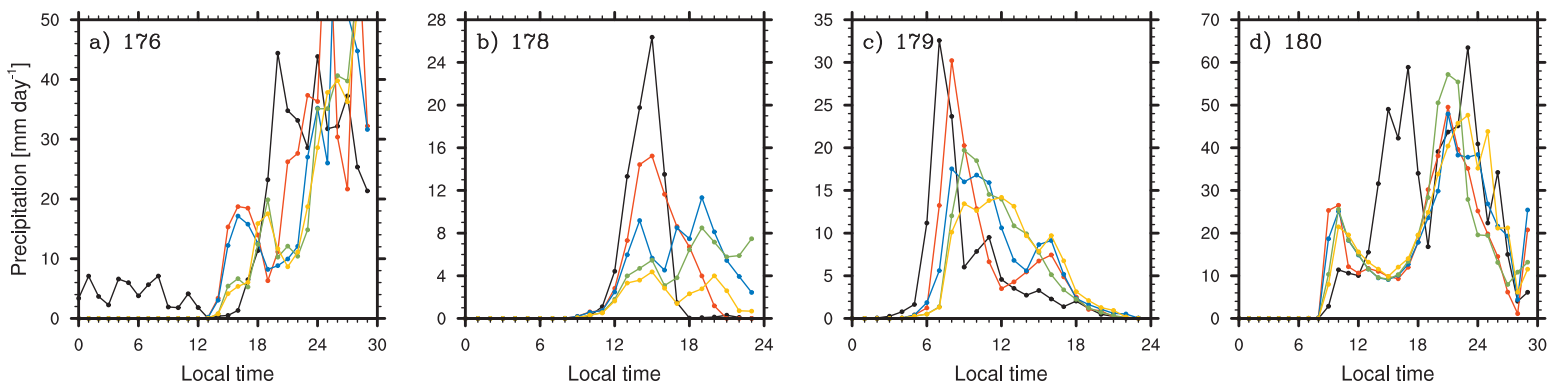

$\longrightarrow$ SAM —UWSDe0sq $\because$ UWSDe0mf $\because$ UWSDall $\because$ UWSDe0

Fig. 13. Diurnal cycle of precipitation for ARM day (a) 176, (b) 178, (c) 179, and (d) 180. Black, blue, green, red and orange lines are for SAM, UWSDe0sq, UWSDe0mf, UWSDall and UWSDe0, respectively.
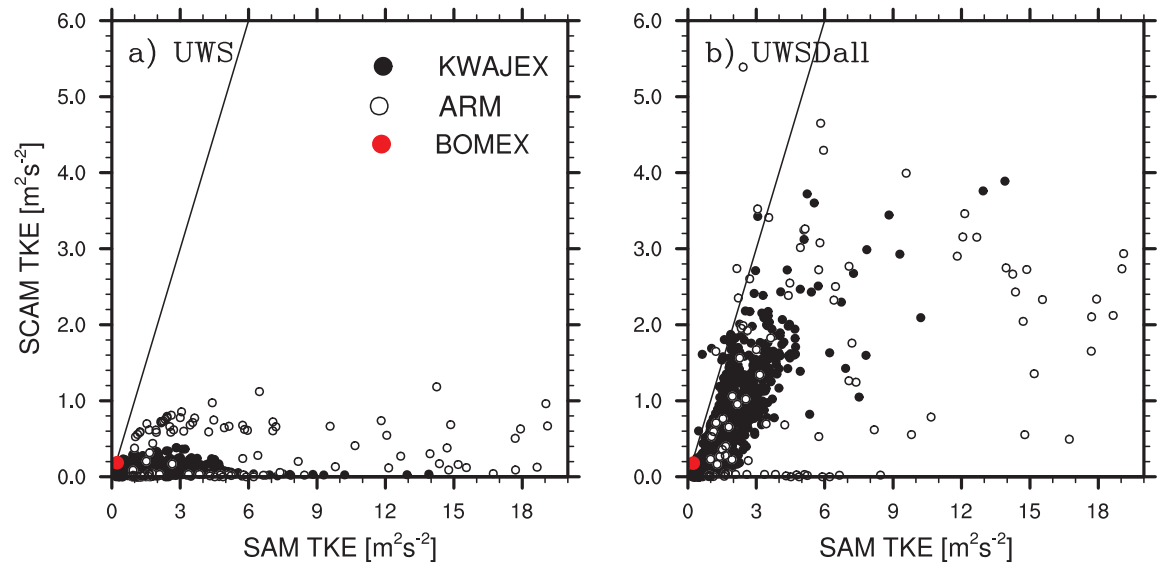

Fig. 14. Scatter plots of PBL averaged TKE in (a) UWS and (b) UWSDall versus SAM values. Black, white and red circles are for KWAJEX, ARM, and BOMEX, respectively. For KWAJEX and ARM, only points with precipitation are plotted. The BOMEX point corresponds to the mean over the simulation hours 3 to 6 . A 1:1 line has also been added to the plots.

convection is precipitation, so that improving the representation of some key effects of precipitation in a shallow convection scheme can allow it to be extended into a unified scheme.

We considered previously studied cases of shallow convection (BOMEX), tropical oceanic convection (KWAJEX) and mid-latitude continental convection (ARM). We used large-eddy simulations of the three cases as benchmarks for parameterization formulation and improvement. We implemented our improved relations in the UW shallow convection scheme and tested the results in the SCAM single-column modeling framework.

We included three main effects of precipitation on convective development, encompassing cloud-base mass flux, cloud-base humidity and entrainment/detrainment rates. Rain evaporation generates cold pools in the PBL, forcing convergence and thus favoring cloud formation. This expresses itself by an increase in boundary-layer TKE, which in the UW scheme is a primary control on cloud-base mass flux. We found that the increase of TKE compared to that in the dry convective boundary layer scales with precipitation at cloud base times the height of the PBL (see Eq. 6). Rain evaporation also modifies the probability distribution function of cloud-base thermodynamic properties, increasing horizontal humidity variance. Cumulus updrafts tend to form over the moister parts of the PBL, so to predict cumulus base humidity we explicitly include a parameterization of humidity variance in terms of cloud-base precipitation rate (see Eq. 7). Finally, the formation of cold pools organizes the planetary boundary layer and the entire cumulus ensemble and indirectly lowers the bulk entrainment rate $\epsilon_{0}$. This effect is represented through a dependence of the cumulus updraft lateral mixing rate on precipitation at cloud base (see Eqs. 9-10). 
These modifications were implemented in the UW shallow convection scheme. In all cases, the new scheme performs as well as or better than the default CAM version. It also outperforms the simulations using the default UW shallow convection scheme as the sole convective parameterization. For our tropical oceanic convection case, the new unified scheme especially improves relative humidity, cloud cover and mass flux profiles. The performance in terms of mid-latitude continental convection is more case-dependent. The main improvement is in the simulated timing of the diurnal cycle when surface fluxes are the dominant forcing for convection. The new unified scheme removes the premature onset of precipitation, which is a common pitfall of deep convective parameterizations, and is able to simulate the peak rainfall rate and duration of rainfall reasonably well. Finally, the scheme can still realistically simulate shallow oceanic trade-cumulus convection.

The main biases, which are present not only with the new scheme but in all of our single-column model experiments, are that the simulated PBL structure tends both to be too well mixed and to insufficiently reduce boundary-layer MSE during deep convection as compared to LES, especially for midlatitude continental convection. We attribute those biases to a combination of two factors. First, to maintain convection, the PBL schemes must sustain a convective PBL that extends from the surface to the convective cloud base. Second, the UW convection scheme does not explicitly consider downdrafts, while the Zhang and McFarlane (1995) scheme only includes saturated downdrafts. Yet most of the downdrafts appear to be unsaturated in the LES.

Of the three tested modifications (i.e., in cloud-base mass flux, cloud-base thermodynamic properties and bulk entrainment rate), changing the bulk updraft lateral mixing rate has the largest impact. Without this, the UW scheme has difficulty in simulating a realistic transition from shallow to deep convection. This is true even though its buoyancy sorting algorithm should allow it to be sensitive to freetropospheric relative humidity and previous cloud-resolving modeling studies (e.g., Chaboureau et al., 2004) have indicated that moistening of the troposphere through detrainment from shallow and/or congestus clouds controls the transition to deep convection. Expressed in other words, precipitation (or its evaporation) is a strong positive feedback in the transition from shallow to deep convection in our single-column model experiments, which helps explain why this transition is rather difficult for cumulus parameterizations to simulate. The impacts of our modifications made to the cloud-base mass flux and cloud-base thermodynamic properties are subtler. Separately, they only have small impacts but taken together, they enhance the sensitivity of convection to prior precipitation and enhance the precipitation peaks. Their inclusion seems especially important for the timing and amplitude of the convective diurnal cycle over mid-latitude continental areas.
All in all our approach does allow for a unified representation of moist convection. It also allows for a representation of the organizational effects of precipitation, which have been shown of importance for convection and are generally not included in convective parameterizations. Finally, it allows for tighter interactions between the planetary boundary layer and convection. Although included in the convection scheme, our modifications directly affect the mean boundary layer properties through the tight coupling produced by the use of a CIN/TKE closure. As indicated in Fletcher and Bretherton (2010), this type of closure maintains the cumulus base near the top of the PBL: an increase in cloud-base mass flux due to cold pool effects will feed back on the height of the PBL, thereby affecting the PBL properties. This is an advance over existing PBL schemes. Our proposed modifications are consistent even without explicitly including a downdraft scheme. Our approach recognizes that cold pools, whether created by subcloud evaporation, or created also by organized convective downdrafts, affect the convective development. Cold pools only require spatially localized rain evaporation in the PBL, not coherent downdrafts descending from high above the PBL top; in fact the downdrafts in tropical marine convection are not very organized or deep.

Our approach may be criticized as quite empirical and biased towards the employed sampled data. As KWAJEX contains many data points and exhibits weak variability, it has the strongest influence on the estimated coefficients. Nevertheless we still considered quite a large data sample and built our different relations on theoretical expectations. The simplicity of the derived relations allows for an easy implementation/testing with other mass flux schemes, as long as such schemes employ a closure related to the PBL state. It also serves as a good proof of concept for our working hypothesis, letting room for more elaborate future refinements. Key unresolved issues remain the formulation of unsaturated downdrafts and a better theoretical foundation for formulating appropriate entrainment/detrainment rates, both issues with which deep convective parameterizations have been struggling for a long time. As a next step, global climate model simulations with CAM will be performed with the new unified scheme.

Acknowledgements. The authors would like to thank Peter Blossey for the SAM simulations. The first author was funded by the Swiss National Science Foundation under the fellowship program for advanced researchers, project PA00P2_124153. Chris Bretherton was funded by NSF grant ATM 0425247 to the CMMAP Science and Technology Center. Comments by the editor and the anonymous reviewers, which help simplifying our relations and improved our manuscript, are gratefully acknowledged.

Edited by: T. J. Dunkerton 


\section{References}

Arakawa, A.: The cumulus parameterization problem: Past, present, and future, J. Climate, 17, 2493-2525, 2004.

Arakawa, A. and Schubert, W. H.: Interaction of a cumulus cloud ensemble with large-scale environment, Part 1, J. Atmos. Sci., 31, 674-701, 1974.

Bechtold, P., Chaboureau, J. P., Beljaars, A., Betts, A. K., Kohler, M., Miller, M., and Redelsperger, J. L.: The simulation of the diurnal cycle of convective precipitation over land in a global model, Q. J. Roy. Meteorol. Soc., 130, 3119-3137, 2004.

Bechtold, P., Kohler, M., Jung, T., Doblas-Reyes, F., Leutbecher, M., Rodwell, J., Vitart, F., and Balsamo, G.: Advances in simulating atmospheric variability with the ECMWF model: From synoptic to decadal time-scales, Q. J. Roy. Meteorol. Soc., 134, 1337-1351, 2008.

Blossey, P. N., Bretherton, C. S., and Cetrone, J.: Cloud-resolving model simulations of KWAJEX: Model sensitivities and comparisons with satellite and radar observations, J. Atmos. Sci., 64, 1488-1508, 2007.

Bretherton, C. S.: Challenges in Numerical Modeling of Tropical Circulations. In The Global Circulation of the Atmosphere, edited by: Schneider, T. and Sobel, A. H., Princeton University Press, USA, 302-330, 2007.

Bretherton, C. S. and Park, S.: A new moist turbulence parameterization in the Community Atmosphere Model, J. Climate, 22, 3422-3448, 2009.

Bretherton, C. S., McCaa, J. R., and Grenier, H.: A new parameterization for shallow cumulus convection and its application to marine subtropical cloud-topped boundary layers. Part I: Description and 1D results, Mon. Weather Rev., 132, 864-882, 2004.

Chaboureau, J. P., Guichard, F., Redelsperger, J. L., and Lafore, J. P.: The role of stability and moisture in the diurnal cycle of convection over land, Q. J. Roy. Meteorol. Soc., 130, 3105-3117, 2004.

Chikira, M. and Sugizama, M.: A cumulus parameterization with state-dependent entrainment rate, Part I: Description and sensitivity to temperature and humidity profiles, J. Atmos. Sci., 67, 2171-2193, 2010.

Collins, W. D., Rasch, P. J., Boville, B. A., Hack, J. J., McCaa, J. R., Williamson, D. L., Briegleb, B. P., Bitz, C. M., Lin, S. J., and Zhang M.: The formulation and atmospheric simulation of the Community Atmosphere Model Version 3 (CAM3), J. Climate, 19, 2144-2161, 2006.

Dai, A. G., Giorgi, F., and Trenberth, K. E.: Observed and modelsimulated diurnal cycles of precipitation over the contiguous United States, J. Geophys. Res., 104, 6377-6402, 1999.

Deardorff, J. W.: Usefulness of liquid-water potential temperature in a shallow-cloud model, J. Appl. Meteor., 15, 98-102, 1976.

Deng, L. P. and Wu, X. Q.: Effects of convective processes on GCM simulations of the Madden-Julian Oscillation, J. Climate, 23, 352-377, 2010.

Emanuel, K. A.: A scheme for representing cumulus convection in large-scale models, J. Atmos. Sci., 48, 2313-2335, 1991.

Fletcher, J. K. and Bretherton, C. S.: Evaluating boundary layerbased mass flux closures using cloud-resolving model simulations of deep convection, J. Atmos. Sci., 67, 2212-2225, 2010.

Grandpeix, J. Y., Lafore, J. P., and Cheruy, F.: A density current parameterization coupled with Emanuel's convection scheme, Part II: 1D simulations, J. Atmos. Sci., 67, 898-922, 2010.
Guichard, F, Petch, J. C., Redelsperger, J. L., Bechtold, P., Chaboureau, J. P., Cheinet, S., Grabowski, W., Grenier, H., Jones, C. G., Kohler, M., Piriou, J. M., Tailleux, R., and Tomasini, M.: Modelling the diurnal cycle of deep precipitating convection over land with cloud-resolving models and singlecolumn models, Q. J. Roy. Meteorol. Soc., 130, 3139-3172, 2004.

Hack, J. J.: Parameterization of moist convection in the National Center for Atmospheric Research Community Climate Model (CCM2), J. Geophys. Res., 99, 5551-5568, 1994.

Hack, J. J. and Pedretti, J. A.: Assessment of solution uncertainties in single-column modeling frameworks, J. Climate, 13, 352-365, 2000.

Holtslag, A. A. M. and Boville, B. A.: Local versus nonlocal boundary layer diffusion in a global climate model, J. Climate, 6, 18251842, 1993.

Kain, J. S.: The Kain-Fritsch convective parameterization: an update, J. Clim. Appl. Meteorol., 43, 170-181, 2004.

Khairoutdinov, M. F. and Randall, D. A.: Cloud Resolving Modeling of the ARM Summer 1997 IOP: Model Formulation, Results, Uncertainties, and Sensitivities, J. Atmos. Sci., 60, 607625, 2003.

Khairoutdinov, M. F. and Randall, D. A.: High-resolution simulation of shallow-to-deep convection transition over land, J. Atmos. Sci., 63, 3421-3436, 2006.

Kuang, Z. and Bretherton, C. S.: A mass-flux scheme view of a high-resolution simulation of a transition from shallow to deep cumulus convection, J. Atmos. Sci., 63, 1895-1909, 2006.

Lee, M. I., Schubert, S. D., Suarez, M. J., Held, I. M., Lau, N. C., Ploshay, J. J., Kumar, A., Kim, H. K., and Schemm, J. K. E: An analysis of the warm-season diurnal cycle over the continental United States and northern Mexico in general circulation models, J. Hydrometeorol., 8, 344-366, 2007.

Li, L. J., Wang., B., Wang, Y. Q., and Wan, H.: Improvements in climate simulation with modifications to the Tiedtke convective parameterization in the grid-point atmospheric model of IAP LASG (GAMIL), Adv. Atmos. Sci., 24, 323-335, 2007.

Lin, J. L.: The double-ITCZ problem in IPCC AR4 coupled GCMs: Ocean-atmosphere feedback analysis, J. Climate, 20, 44974525, 2007.

Mapes, B.E and Neale, R. B.: Parameterizing convective organization, J. Adv. Model. Earth Syst., 3, M06004, 2011.

Neale, R., Richter, J. R., and Jochum, M.: The impact of convection on ENSO: From a delayed oscillator to a series of events, J. Climate, 21, 5904-5924, 2008.

Neggers, R. A. J., Siebesma, A. P., and Jonker H. J. J.: A multiparcel model for shallow cumulus convection, J. Atmos. Sci., 16551668, 2002.

Park, S. and Bretherton, C. S.: The University of Washington shallow convection and moist turbulence schemes and their impact on climate simulations with the Community Atmosphere Model, J. Climate, 22, 3449-3469, 2009.

Plant, R. S.: A review of the theoretical basis for bulk mass flux convective parameterization, Atmos. Chem. Phys., 10, 3529-3544, doi:10.5194/acp-10-3529-2010, 2010.

Randall, D. A., Khairoutdinov, M., Arakawa, A., and Grabowski, W.: Breaking the cloud parameterization deadlock, B. Am. Meteorol. Soc., 84, 1547-1564, 2003.

Richter, J. R. and Rasch, P. J.: Effects of convective momentum 
transport on the atmospheric circulation in the Community Atmospheric Model, version 3 (CAM3), J. Climate, 21, 14871499, 2008.

Rio, C., Hourdin, F., Grandpeix, J. Y., and Lafore, J. P.: Shifting the diurnal cycle of parameterized deep convection over land, Geophys. Res. Lett., 36, L07809, doi:10.1029/2008GL036779, 2009.

Siebesma, A. P., Bretherton, C. S., Brown, A., Chlond, A., Cuxart, J., Duynkerke, P. G., Jiang, H., Khairoutdinov, M., Lewellen, D., Moeng, C. H., Sanchez, E., Stevens, B., and Stevens, D. E. : A large-eddy simulation intercomparison study of shallow cumulus convection, J. Atmos. Sci., 60, 1201-1219, 2003.

Slingo, J. M., Sperber, K. R., Boyle, J. S., Ceron, J. P., Dix, M., Dugas, B., Ebisuzaki, W., Fyfe, J., Gregory, D., Gueremy, J. F., Hack, J., Harzallah, A., Inness, P., Kitoh, A., Lau, W. K. M., McAvaney, B., Madden, R.,Matthews, A., Palmer, T. N., Park, C. K., Randall, R., and Renno, N.: Intraseasonal oscillations in 15 atmospheric general circulation models: Results from an AMIP diagnostic subproject, Clim. Dynam., 12, 325-257, 1996.
Wang, Y. Q., Zhou, L., and Hamilton, K.: Effect of convective entrainment/detrainment on the simulation of the tropical precipitation diurnal cycle, Mon. Weather Rev., 135, 567-585, 2007.

Yang, G. Y. and Slingo, J.: The diurnal cycle in the Tropics, Mon. Weather Rev., 129, 784-801, 2001.

Zhang, B. J. and McFarlane, N. A.: Sensitivity of climate simulations to the parameterization of cumulus convection in the Canadian Climate Centre general circulation model, Atmos.-Ocean, 33, 407-446, 1995.

Zhang, G. J. and Mu, M. Q.: Simulation of the Madden-Julian oscillation in the NCAR CCM3 using a revised Zhang-McFarlane convection parameterization scheme, J. Climate, 18, 4046-4064, 2005. 